\title{
BRIEF COMMUNICATION OPEN Clinical and radiographic response following targeting of BCAN-NTRK1 fusion in glioneuronal tumor
}

Christopher Alvarez-Breckenridge ${ }^{1}$, Julie J. Miller ${ }^{2}$, Naema Nayyar ${ }^{2,3}$, Corey M. Gill ${ }^{3}$, Andrew Kaneb ${ }^{3}$, Megan D'Andrea ${ }^{3}$, Long P. Le ${ }^{4}$, Jesse Lee ${ }^{4}$, Ju Cheng ${ }^{4}$, Zongli Zheng ${ }^{4}$, William E. Butler ${ }^{1}$, Pratik Multani ${ }^{5}$, Edna Chow Maneval ${ }^{5}$, Sun Ha Paek ${ }^{6}$, Brian D. Toyota ${ }^{7}$, Dora Dias-Santagata ${ }^{4}$, Sandro Santagata ${ }^{8}$, Javier Romero ${ }^{9}$, Alice T. Shaw ${ }^{3}$, Anna F. Farago ${ }^{3}$, Stephen Yip ${ }^{10}$, Daniel P. Cahill ${ }^{1}$, Tracy T. Batchelor ${ }^{2,11,12}$, A. John lafrate ${ }^{4}$ and Priscilla K. Brastianos ${ }^{2,3}$

Glioneuronal tumors constitute a histologically diverse group of primary central nervous system neoplasms that are typically slowgrowing and managed conservatively. Genetic alterations associated with glioneuronal tumors include BRAF mutations and oncogenic fusions. To further characterize this group of tumors, we collected a cohort of 26 glioneuronal tumors and performed indepth genomic analysis. We identified mutations in BRAF (34\%) and oncogenic fusions (30\%), consistent with previously published reports. In addition, we discovered novel oncogenic fusions involving members of the NTRK gene family in a subset of our cohort. One-patient with BCAN exon 13 fused to NTRK1 exon 11 initially underwent a subtotal resection for a 4th ventricular glioneuronal tumor but ultimately required additional therapy due to progressive, symptomatic disease. Given the patient's targetable fusion, the patient was enrolled on a clinical trial with entrectinib, a pan-Trk, ROS1, and ALK (anaplastic lymphoma kinase) inhibitor. The patient was treated for 11 months and during this time volumetric analysis of the lesion demonstrated a maximum reduction of $60 \%$ in the contrast-enhancing tumor compared to his pre-treatment magnetic resonance imaging study. The radiologic response was associated with resolution of his clinical symptoms and was maintained for 11 months on treatment. This report of a BCAN-NTRK1 fusion in glioneuronal tumors highlights its clinical importance as a novel, targetable alteration.

npj Precision Oncology (2017)1:5; doi:10.1038/s41698-017-0009-y

\section{INTRODUCTION}

Glioneuronal tumors are a collection of uncommon, diverse primary central nervous system (CNS) neoplasms that exhibit variable degrees of glial and neuronal differentiation. The prognosis for patients with these tumors is generally favorable due to their non-infiltrative, well-circumscribed, and surgically accessible features. However, a subset of these patients have nonresectable disease or tumors that take on an unusually aggressive course, necessitating treatment with either radiation or chemotherapy, which both have limited efficacy. Glioneuronal tumors are histologically, genetically, and clinically diverse. Advances in the molecular characterization of CNS tumors, particularly in primitive neuroectodermal tumors of the central nervous system ${ }^{1}$ and low grade gliomas, ${ }^{2}$ have provided a blueprint for creating a similar molecular framework for classification of histologically heterogeneous glioneuronal tumors. In addition to refining the classification and diagnosis of a previously diverse collections of tumors, recent advances in molecular profiling has aided in the identification of novel oncogenic drivers and targetable alterations leading to expansion of targeted therapies across various cancer. These include targeting EGFR mutations and oncogenic $A L K$ fusions in NSCLC with tyrosine kinase inhibitors, ${ }^{3,} 4$ and oncogenic BRAF mutations in melanoma and NSCLC with dabrafenib and trametinib. ${ }^{5}$ Thus, in the modern era, genomic characterization and drug development often progress in parallel to facilitate the rapid evaluation of novel pharmaceutical agents against newly identified, putative oncogenic drivers. ${ }^{6}$

\section{RESULTS}

To explore the diversity of genetic alterations in glioneuronal tumors, a cohort of 26 tumors with pathologic diagnoses that included glioneuronal tumor or ganglioglioma was collected (15 from Massachusetts General Hospital, Boston, 11 from Vancouver General Hospital, Vancouver), and examined for BRAF V600 and IDH1 mutations and oncogenic fusions using targeted next generation sequencing (NGS). ${ }^{7}$ BRAF V600E and IDH1 R132H mutant protein expression was confirmed by immunohistochemistry. As expected, we identified several recurrent BRAF V600E mutations (9 of 26), consistent with previous reports. ${ }^{8}$ In addition,

\footnotetext{
${ }^{1}$ Department of Neurosurgery, Massachusetts General Hospital, Harvard Medical School, Boston, MA, USA; ${ }^{2}$ Department of Neurology, Massachusetts General Hospital, Harvard Medical School, Boston, MA, USA; ${ }^{3}$ Department of Medicine, Massachusetts General Hospital, Harvard Medical School, Boston, MA, USA; ${ }^{4}$ Department of Pathology, Massachusetts General Hospital, Harvard Medical School, Boston, MA, USA; ${ }^{5}$ Ignyta, Inc., San Diego, CA, USA; ${ }^{6}$ Department of Neurosurgery, Seoul National University Hospital, Seoul, Korea; ${ }^{7}$ Division of Neurosurgery, Department of Surgery, University of British Columbia, Vancouver, BC, Canada; ${ }^{8}$ Department of Pathology, Division of Neuropathology, Brigham \& Women's Hospital, Harvard Medical School, Boston, MA, USA; ${ }^{9}$ Department of Radiology, Massachusetts General Hospital, Harvard Medical School, Boston, MA, USA;

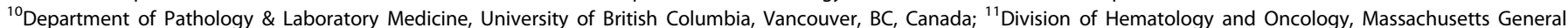
Hospital, Harvard Medical School, Boston, MA, USA and ${ }^{12}$ Department of Radiation Oncology, Massachusetts General Hospital, Harvard Medical School, Boston, MA, USA Correspondence: Priscilla K. Brastianos (pbrastianos@mgh.harvard.edu)

Christopher Alvarez-Breckenridge and Julie J. Miller contributed equally to this work

Tracy T. Batchelor, A. John lafrate and Priscilla K. Brastianos jointly supervised this work
}

Received: 6 October 2016 Revised: 5 December 2016 Accepted: 30 December 2016

Published online: 20 March 2017 
Table 1. Molecular alterations found in glioneuronal tumors

\begin{tabular}{|c|c|c|c|c|}
\hline Histologic diagnosis & Who grade & Age at diagnosis & Fusions & Braf V600E mutation \\
\hline Glioneuronal tumor & 1 & 31 & KIAA1549 ex16-BRAF ex9 & No \\
\hline Glioneuronal tumor & & 32 & None & No \\
\hline Glioneuronal tumor & & 26 & EWSR1 ex9-PATZ1 ex1 & ND \\
\hline Low-grade glioneuronal tumor & & 18 & None & ND \\
\hline Low-grade glioneuronal tumor & & 15 & FGFR1 ex18-TACC1 ex7 & No \\
\hline Low-grade glioneuronal tumor & & 30 & None & Yes \\
\hline Low-grade glioneuronal tumor & & 29 & None & Yes \\
\hline Low-grade glioneuronal tumor & & 19 & None & No \\
\hline Low-grade glioneuronal tumor & & 74 & None & No \\
\hline Ganglioglioma & 1 & 20 & None & Yes \\
\hline Ganglioglioma & 1 & 33 & STRN3 ex7-NTRK2 ex16 & No \\
\hline Ganglioglioma & 1 & 39 & PRKAR2B ex1-BRAF ex10 & No \\
\hline Ganglioglioma & 1 & 70 & None & No \\
\hline Composite ganglioglioma/Pilocytic astrocytoma & 1 & 24 & None & Yes \\
\hline Anaplastic ganglioglioma & & 24 & None & Yes \\
\hline Atypical ganglioglioma & II & 33 & None & No \\
\hline Composite DNT and ganglioglioma & & 29 & None & Yes \\
\hline Composite ganglioglioma and DNT & 1 & 23 & None & Yes \\
\hline
\end{tabular}

we identified known and novel fusions of FGFR1-TACC1, KIAA1549BRAF, PATZ1-EWSR1, PRKAR2B-BRAF, STRN3-NTRK2, WNK2-NTRK2, and BCAN-NTRK1 (8 of 26) (Table 1). Of note, three tumors in our cohort contained a fusion involving the neurotrophic tropomyosin receptor kinase gene family (NTRK), which encode the Trk transmembrane receptors. Fusions involving the NTRK family have been reported in a number of different cancers and lead to constitutive activation of Trk protein kinase activity. ${ }^{9}, 10$

Consistent with recent reports documenting targetable NTRK fusions in other cancers, including a small percentage of patients with non-small cell lung cancer, ${ }^{11}$ our finding of a BCAN-NTRK1 fusion in a glioneuronal patient raised the possibility of therapeutic intervention. A 54-year-old man with a tumor containing this fusion initially underwent a resection of the symptomatic, enlarging 4th ventricular mass. Due to the lowgrade features of the tumor and its proximity to the medulla, a subtotal resection, freeing entry of the cerebral aqueduct was performed.

Findings from the permanent pathologic specimen were notable for a low cellularity tumor in a densely fibrillary background, numerous Rosenthal fibers and eosinophilic granular bodies, and a low Ki-67 labeling index ( $3 \%)$. The tumor included a heterogeneous population of GFAP and synaptophysin immunopositive cells and did not stain for either NeuN or IDH1 R132H. These collective findings were felt to be consistent with a mixed low-grade glioneuronal tumor with pilocytic features. Following a period of clinical and radiologic stability, an magnetic resonance imaging (MRI) performed 3 years after the surgery revealed interval growth in the lesion with associated mass effect on the pons. Given the patient's indolent symptom of diplopia, mild progression on imaging and reluctance for radiotherapy, targeted pharmacological therapy options were considered. Targeted NGS was performed on RNA extracted from the tumor and uncovered a fusion (confirmed by FISH, Fig. 1a), involving BCAN exon 13 fused to NTRK exon 11, including an intact and in-frame tyrosine kinase domain of TrkA (Fig. 1b). He enrolled on a phase 1 dose-escalation clinical trial of entrectinib (RXDX-101), a pan-Trk, ROS1, and ALK oral tyrosine kinase inhibitor (ClinicalTrials.gov Identifier: NCT02097810). Entrectinib was recommended given previous reports of clinical activity in tumors harboring NTRK gene fusions in colorectal carcinoma and non-small cell lung cancer ${ }^{11,12}$ as well as for the demonstrated activity of entrectinib to penetrate the central nervous system. ${ }^{11}$ The patient received an entrectinib dose of $600 \mathrm{mg}$ orally each day and experienced lower extremity edema as the only documented side effect of the medication.

After 9 months of therapy, volumetric analysis of the lesion demonstrated a $60 \%$ reduction in contrast-enhancing tumor compared to his pre-treatment MRI (Fig. 1c, d). This radiographic improvement was accompanied by improvement of diplopia, which incidentally worsened with temporary cessation of entrectinib for 5 days during his treatment course. Two months later, however, the patient developed worsening diplopia and imaging of the lesion demonstrated a gradual interval increase in size. Therefore, in light of radiographic progression and worsening symptoms, the decision was made to discontinue entrectinib after 11 cycles and refer the patient for radiotherapy.

\section{DISCUSSION}

In our cohort of 26 glioneuronal tumors, the prevalence of BRAF pathway activation (34\% patients with mutations, $7 \%$ with fusion) and gene fusions involving other oncogenes ( $30 \%$ of patients) is 
A.

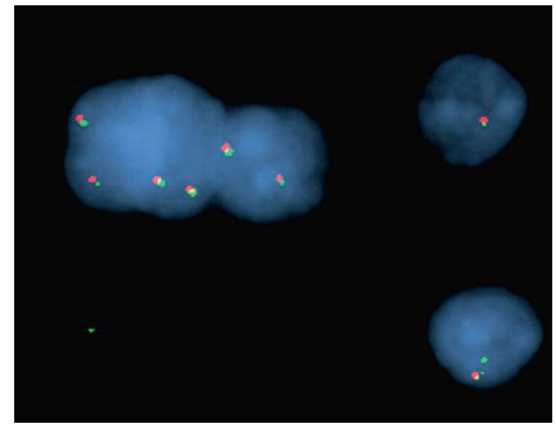

B.
$B C A N$ exons $1-13$
NTRK1 exons 16-22

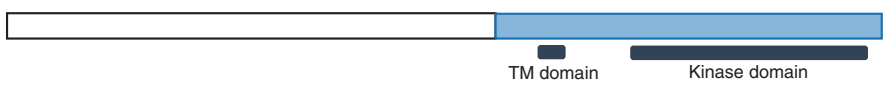

NTRK2 exons 16-21

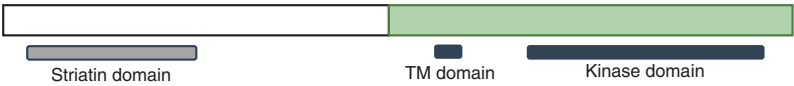

WNK2 exons 1-24

NTRK2 exons 16-21

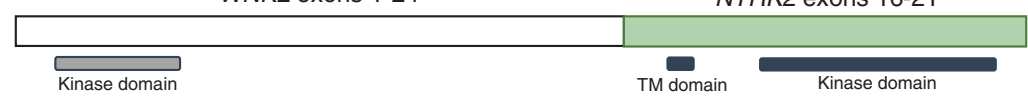

C.

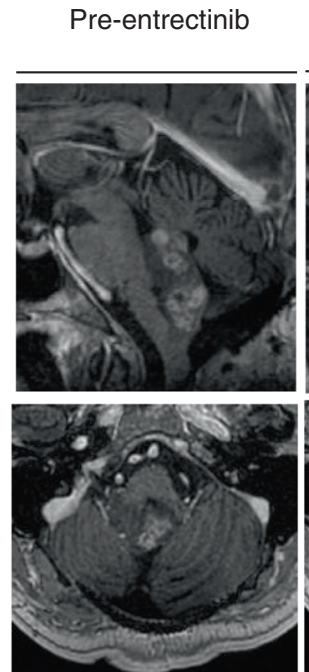

9 months on treatment

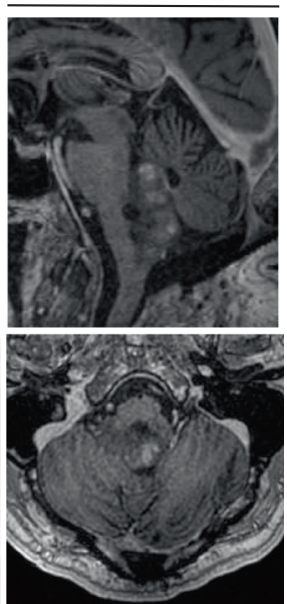

D.

\section{Change in tumor volume}

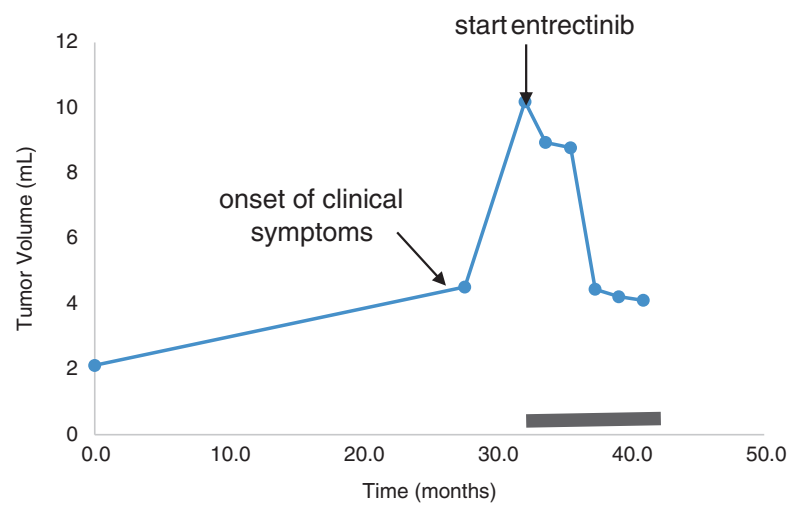

Fig. 1 NTRK fusion in glioneuronal tumors can be treated with Trk-inhibitors. a Fluorescence in situ hybridization (FISH) using split apart probes, with separation of the $5^{\prime}$ (green) and $3^{\prime}$ (red) NTRK1 signals, reveal abnormal rearrangement, with some red-green pairs showing a small green probe signal (arrows). b Schematic of three different NTRK-containing gene fusions discovered in the glioneuronal cohorts from MGH and Vancouver, involving either NTRK1 or NTRK2. Predicted active domains in the expression product are depicted below. TM transmembrane. c Sagittal (top) and axial (bottom) post-contrast T1-weighted MRI images of patient with BCAN-NTRK1 fusion just prior to treatment with entrectinib (left) and following 9 months on treatment (right). The T1-avid tumor visualized in dorsal pons and medulla has decreased in size during this time period. d Plot demonstrating tumor volume over time while patient was on treatment with entrectinib using MRI-derived volumetrics (see Methods). Baseline tumor volume was measured approximately 2 years prior to treatment. Tumor volume initially slowly increased, then expanded more rapidly coincident with onset of clinical symptoms. Treatment with entrectinib led to rapid and substantial decrease in tumor volume. Period on treatment denoted with thick black line

consistent with recently published results across a series of lowgrade neuroepithelial tumors in children, which found BRAF alterations in 9 of 17 gangliogliomas (including $B R A F$ V600E mutation, MACF1-BRAF, AGK-BRAF, and GNAI1-BRAF fusions). ${ }^{13}$ In common with our cohort, Qaddoumi et al. also observed fusions in EWSR1-PATZ1 and SLMAP-NTRK2 in 2 out of 17 gangliogliomas. ${ }^{13}$ Moreover, fusions incorporating FGFR1-TACC1 and BRAF-RNF130 have been reported in dysembryoplastic neuroepithelial tumor and diffuse oligodendroglial tumors, and KIAA1549-BRAF has been established as a driver in infratentorial pilocytic astrocytomas. ${ }^{13}$ These results suggest that BRAF alterations and oncogenic fusions are key drivers in glioneuronal pathogenesis and represent a potential target for molecularly guided therapy. In the setting of glioneuronal tumors, a variety of genetic alterations, each occurring at relatively low frequency, appear to contribute to their development. Our finding of BCAN-NTRK1, STRN3-NTRK2 and WNK2-NTRK2 fusions highlight NTRK-related fusions as a recurrent alteration in glioneuronal tumors.

Fusions involving NTRK1, NTRK2, and NTRK3 also have been reported to occur at a low frequency across multiple tumor types. ${ }^{14}$ Analysis of The Cancer Genome Atlas shows NTRK1,
NTRK2, and NTRK3 fusions with concomitant oncogenic activation in multiple signaling pathways, such as MAPK and AKT, across a variety of tumors. ${ }^{15}$ Further, Jones et al. report recurrent NTRK2 fusions in pediatric pilocytic astrocytomas ${ }^{16}$ and Kim et al. report a BCAN-NTRK1 in glioblastoma. ${ }^{17}$ Based on the results from our study, we propose that NTRK fusions are novel oncogenic events that similarly serve as actionable targets.

The utility of targeting NTRK1 was first described by Vaishnavi et $a$ l. in lung cancer where the authors identified oncogenic fusions involving MPRIP-NTRK1 and CD74-NTRK1, leading to constitutive activation of the kinase domain of the NTRK1 expression product, TrkA. ${ }^{18}$ The relevance of this finding was demonstrated in 3 of 91 lung cancer patients with newly diagnosed NTRK1 fusions. Targeting these fusions with ARRY470, CEP-701, and crizotinib, which inhibit autophosphorylation of MPRIP-NTRK1 and CD74-NTRK1, led to inhibited proliferation and colony formation, and induced cell cycle arrest, ${ }^{18}$ validating the oncogenicity of these gene fusions.

The utility of this approach was further highlighted in the context of LMNA-NTRK1 fusions in soft tissue sarcoma, congenital infantile fibrosarcoma (CIFS), and colorectal cancer. ${ }^{12,}{ }^{19-21}$ In the 
setting of metastatic soft tissue sarcoma of the thigh, targeting the lamin A/C (LMNA) and NTRK1 fusion with the TrkA inhibitor, LOXO101 , led to a rapid clinical, radiographic, and serologic response. ${ }^{19}$ Similarly, an infant with metastatic CIFS was found to have an LMNA-NTRK1 fusion in addition to biallelic losses of CDKN2A and $C D K N 2 B$. The child was started on crizotinib and after 6 weeks of treatment demonstrated regression of metastatic disease. ${ }^{21}$

The LMNA-NTRK1 fusion has similarly been demonstrated in colorectal cancer. ${ }^{12,} 20$ In a study by Sartore-Bianchi et al. a patient with primary colon cancer, peritoneal carcinomatosis, and liver metastases was similarly found to have a LMNA-NTRK1 fusion. Treatment with entrectinib was initiated, resulting in a partial response with decrease in the size of multiple metastatic lesions. ${ }^{20}$ However, the patient, who was being treated on an intermittent dosing schedule during the early stages of entrectinib dose finding, ultimately developed disease progression in the setting of treatment resistance. Interestingly, Russo and colleagues noted that, at the time of tumor progression, circulating tumor DNA was found to have two novel NTRK1 mutations (NTRK1, p.G595R and p.G667C) that were not detectable in the plasma at the initiation of therapy, demonstrating evidence of acquired resistance to entrectinib. $^{20}$

In our study, we expand upon the recent success of targeting NTRK fusions across various cancer types by reporting the first treatment of a glioneuronal tumor with a pan-Trk inhibitor. This treatment was associated with a radiographic and clinical response for a sustained period of time. Thus, our results underscore the importance of examining newly diagnosed glioneuronal tumors for fusions, while also emphasizing the need for ongoing drug development to target these novel oncogenic fusions. These results emphasize the value of identifying unique molecular subpopulations of patients with low-frequency genomic alterations. ${ }^{6}$ More generally, our findings highlight the need for a tailored approach to oncologic care in which patient samples are examined for unique molecular drivers that can ultimately be treated with emerging targeted therapies. This work also highlights the need for CNS penetrant compounds in order to effectively treat primary CNS neoplasms as well as other solid tumors with a propensity to metastasize to the brain.

\section{METHODS}

Gene fusion assay

The Anchored Multiplex PCR for targeted fusion transcript detection using NGS was used, as previously described. ${ }^{7}$ Briefly, total nucleic acid was isolated from a formalin-fixed paraffin embedded tumor specimen after histological review for tumor enrichment. The total nucleic acid was reverse transcribed with random hexamers, followed by second strand synthesis to create double-stranded complementary DNA (cDNA). The doublestranded cDNA was end-repaired, adenylated, and ligated with a half-functional adapter. Two hemi-nested PCR reactions were applied to create a fully functional sequencing library that targets specific genes (exons) listed below. Illumina MiSeq $2 \times 147$ base pair paired-end sequencing results were aligned to the hg19 human genome reference using bwa-mem. ${ }^{22}$ A laboratorydeveloped algorithm was used for fusion transcript detection and annotation. The integrity of the input nucleic acid and the technical performance of the assay were assessed with a qualitative reverse transcription $\mathrm{qPCR}$ assay and assessing the DNA/RNA content in the sequencing results. The assay is validated for samples showing $20 \%$ or higher tumor cellularity. FISH was performed on a $5-\mu$ formalin-fixed paraffin embedded tumor section, pretreated with xylene and standard protease and detergent treatment. BAC probe RP11 -1047J23 (5' NTRK1) was labeled green and RP11 -1038N13 (3' NRTK1) red. Images were captured with an Olympus BX61 fluorescence microscope and equipped with a Leica Cytovision workstation.

\section{Volumetric analysis}

Volumetric analysis has been shown to be a sensitive indicator of tumor growth, particularly in tumors with complex shape or slow growth, and is widely used for monitoring changes in nervous system tumors. ${ }^{23,} 24$ Routine MRI containing standard imaging sequences, including T2-, FLAIR-, and T1-weighted sequences were obtained before and after administration of gadolinium. Volumetric measurements were performed with semi-automated outline on $3 \mathrm{~mm}$ T1-weighted post-contrast images. Vitrea, Vital Images, Minnetonka, Minnesota USA. ${ }^{23-26}$

\section{COMPETING INTERESTS}

A.J.I., L.P.L. and Z.Z. own equity in ArcherDx a license of the AMP technology.

\section{REFERENCES}

1. Sturm, D. et al. New brain tumor entities emerge from molecular classification of CNS-PNETs. Cell 164, 1060-1072, doi:10.1016/j.cell.2016.01.015 (2016).

2. Brat, D. J. et al. Comprehensive, integrative genomic analysis of diffuse lowergrade gliomas. N. Engl. J. Med. 372, 2481--2498., doi:10.1056/NEJMoa1402121 (2015).

3. Lynch, T. J. et al. Activating mutations in the epidermal growth factor receptor underlying responsiveness of non-small-cell lung cancer to gefitinib. N. Engl. J. Med. 350, 2129-2139, doi:10.1056/NEJMoa040938 (2004).

4. Kwak, E. L. et al. Anaplastic lymphoma kinase inhibition in non-small-cell lung cancer. N. Engl. J. Med. 363, 1693-1703, doi:10.1056/NEJMoa1006448 (2010).

5. Chapman, P. B. et al. Improved survival with vemurafenib in melanoma with BRAF V600E mutation. N. Engl. J. Med. 364, 2507-2516, doi:10.1056/NEJMoa1 103782 (2011).

6. Okimoto, R. A. \& Bivona, T. G. Tracking down response and resistance to TRK Inhibitors. Cancer Discov. 6, 14-16, doi:10.1158/2159-8290.CD-15-1352 (2016).

7. Zheng, Z. et al. Anchored multiplex PCR for targeted next-generation sequencing. Nat. Med. 20, 1479-1484, doi:10.1038/nm.3729 (2014).

8. Zhang, J. et al. Whole-genome sequencing identifies genetic alterations in pediatric low-grade gliomas. Nat. Genet. 45, 602-612, doi:10.1038/ng.2611 (2013).

9. Amatu, A., Sartore-Bianchi, A. \& Siena, S. NTRK gene fusions as novel targets of cancer therapy across multiple tumour types. ESMO Open 1, e000023 (2016).

10. Alassiri, A. H. et al. ETV6-NTRK3 is expressed in a subset of ALK-negative inflammatory myofibroblastic tumors. Am. J. Surg. Pathol. 40, 1051-1061, doi:10.1097/PAS.0000000000000677 (2016).

11. Farago, A. F. et al. Durable clinical response to entrectinib in NTRK1-rearranged non-small cell lung cancer. J. Thorac. Oncol. 10, 1670-1674, doi:10.1097/01. JTO.0000473485.38553.fO (2015).

12. Sartore-Bianchi, A. et al. Sensitivity to entrectinib associated with a novel LMNANTRK1 gene fusion in metastatic colorectal cancer. J. Natl Cancer Inst. 108, doi:10.1093/jnci/djv306 (2016).

13. Qaddoumi, I. et al. Genetic alterations in uncommon low-grade neuroepithelial tumors: BRAF, FGFR1, and MYB mutations occur at high frequency and align with morphology. Acta Neuropathol. 131, 833-845, doi:10.1007/s00401-016-1539-z (2016).

14. Vaishnavi, A., Le, A. T. \& Doebele, R. C. TRKing down an old oncogene in a new era of targeted therapy. Cancer Discov. 5, 25-34, doi:10.1158/2159-8290.CD-14-0765 (2015).

15. Stransky, N., Cerami, E., Schalm, S., Kim, J. L. \& Lengauer, C. The landscape of kinase fusions in cancer. Nat. Commun. 5, 4846, doi:10.1038/ncomms5846 (2014).

16. Jones, D. T. et al. Recurrent somatic alterations of FGFR1 and NTRK2 in pilocytic astrocytoma. Nat. Genet. 45, 927-932, doi:10.1038/ng.2682 (2013).

17. Kim, J. et al. NTRK1 fusion in glioblastoma multiforme PLoS One 9, e91940, doi:10.1371/journal.pone.0091940 (2014).

18. Vaishnavi, A. et al. Oncogenic and drug-sensitive NTRK1 rearrangements in lung cancer. Nat. Med. 19, 1469-1472, doi:10.1038/nm.3352 (2013).

19. Doebele, R. C. et al. An oncogenic NTRK Fusion in a patient with soft-tissue sarcoma with response to the tropomyosin-related kinase inhibitor LOXO-101. Cancer Discov. 5, 1049-1057, doi:10.1158/2159-8290.CD-15-0443 (2015).

20. Russo, M. et al. Acquired resistance to the TRK inhibitor entrectinib in colorectal cancer. Cancer Discov. 6, 36-44, doi:10.1158/2159-8290.CD-15-0940 (2016). 
21. Wong, V. et al. Evaluation of a congenital infantile fibrosarcoma by comprehensive genomic profiling reveals an LMNA-NTRK1 gene fusion responsive to Crizotinib. J. Natl Cancer Inst. 108, doi:10.1093/jnci/djv307 (2016).

22. Li, H. \& Durbin, R. Fast and accurate short read alignment with Burrows-Wheele transform. Bioinformatics 25, 1754-1760, doi:10.1093/bioinformatics/btp324 (2009).

23. Dombi, E. et al. Recommendations for imaging tumor response in neurofibromatosis clinical trials. Neurology 81, S33-40 (2013).

24. Plotkin, S. R. et al. Hearing improvement after bevacizumab in patients with neurofibromatosis type 2. N. Engl. J. Med. 361, 358-367, doi:10.1056/ NEJMoa0902579 (2009).

25. Brastianos, P. K. et al. Dramatic response of BRAF V600E mutant papillary craniopharyngioma to targeted therapy. J. Natl Cancer Inst. 108, doi:10.1093/jnci/ djv310 (2016).
26. Shah, G. D. et al. Comparison of linear and volumetric criteria in assessing tumor response in adult high-grade gliomas. Neuro-oncology 8, 38-46 (2006).

(i) This work is licensed under a Creative Commons Attribution 4.0 International License. The images or other third party material in this article are included in the article's Creative Commons license, unless indicated otherwise in the credit line; if the material is not included under the Creative Commons license, users will need to obtain permission from the license holder to reproduce the material. To view a copy of this license, visit http://creativecommons.org/licenses/by/ $4.0 /$

(c) The Author(s) 2017 